\title{
AXIOLOGISATION OF CONTEMPORARY CONCEPTS AND TERMS - ON THE EXAMPLE OF CLIMATE
}

\author{
ILIANA GENEW-PUHALEWA \\ UNIVERSITY OF WARSAW \\ i.genew-puhalewa@uw.edu.pl
}

The paper discusses the issue of axiologisation of concepts that originated in the field of science as axiologically neutral. The subject links the study with the subdiscipline known as linguistic axiology (or axiolinguistics), which has made significant achievements in Slavic studies in the last decades. Concepts and terms of the thematic area 'Environment' (e.g. environment, ecological, sustainability, eco-, recycling, environmental impact) are considered as suitable for observing semantic development that involves evaluative connotation. The author analyses thoroughly the concept of CLIMATE through the corresponding terms and its derivatives. The need to update lexicographical descriptions of the terms expressing contemporary ideas and values has been emphasised.

Due to the global reach of the phenomena discussed, it is proposed that the arguments concerning Bulgarian can be applied to other European languages.

Keywords: axiologisation, value, term, climate, climate change, environment, Bulgarian

\section{REFERENCES}

Apresian 1995: Apresian, Iu. D. Integral'noe opisanie iazyka i sistemnaia leksikografiia [Integral Language Description and Systematic Lexicography]. Izbran- nye trudy, t. 2. Moskva, Shkola "Iazyki russkoi kul'tury".

Arutiunova 1988: Arutiunova, N. D. Tipy iazykovyh znachenii. Ocenka. Sobytie, Fakty [Types of Language Values. Evaluation. Event. Fact]. Moskva, Nauka.

BTR 1994: Popov, D. (ed.) Balgarski talkoven rechnik [Bulgarian Explanatory Dictionary]. Sofia, Nauka i izkustvo.

Bartminski 1991: Bartminski, J. Projekt i zalozenia ogólne slownika aksjologicznego, „Jçzyk a Kultura“, t. 2: Zagadnienia leksykalne i aksjologiczne, red. J. Puzynina, J. Bartminski. Wroclaw, Uniwersytet Wroclawski, s. 197-209.

Bartminski 2006: Bartminski, J. Jçzykowe podstawy obrazu swiata. Lublin, Wydawnictwo Uniwersytetu Marii Curie-Sklodowskiej.

Bartminski 2015: Bartminski, J. Leksykon aksjologiczny Slowian i ich sqsiadów co zawiera, na jakich zasadach siç opiera, dla kogo jest przeznaczony? - In: Leksykon aksjologiczny Slowian i ich sqsiadów. Tom 1: Dom. Lublin, Wydawnictwo Uniwersytetu Marii Curie-Sklodowskiej, s. 7-13. 
Bartminski, Grzeszczak 2014: Bartminski, J., M. Grzeszczak. Jak rekonstruowac kanon wartosci narodowych i europejskich? „Etnolingwistyka“, 26. Lublin, s. 21-44.

Dlugosz 2017: Dlugosz, N. O znakach ubezwlasnowolnionych czyli o nowych polskich i bulgarskich compositach bezafiksalnych w medialnym dyskursie publicystycznym (ujçcie kognitywno-komunikacyjne). Poznan, Wydawnictwo Naukowe UAM.

Gaberov 2000: Kolektiv. Balgarski entsiklopedichen rechnik A-Z [Bulgarian Encyclopedic Dictionary A-Z].Veliko Tarnovo, Gaberov.

Genew-Puhalewa 2014: Genew-Puhalewa, I. Za prilagatelnoto ime ,ekologichen“ kato terminoelement [On the Adjective екологичен as a Term Element]. - Balgarski ezik, LXI, № 1, s. 89-95.

Genew-Puhalewa 2015: Genew-Puhalewa, I. Terminologiyata na Evropeyskiya sayuz. Sapostavka na balgarskata, gratskata, polskata i angliyskata terminologiya na pravoto na okolnata sreda [Comparative Terminology of the EU Acquis. Comparative Analysis of Bulgarian, Greek, Polish and English Terminology in the Field of Environmental Law]. Katowice, Wydawnictwo Uniwersytetu Sląskiego.

Genew-Puhalewa 2018: Genew-Puhalewa, I. Slavyanski saotvetsviya na angliyskiya termin sustainability [Slavonic Equivalents to the English Term Sustainability]. - Studia z filologiipolskiej i slowianskiej, № 8, s. 260-272.

Genew-Puhalewa, Sotirov 2017: Genew-Puhalewa, I., P. Sotirov. Proektyt EURO JOS na Polskata akademiya na naukite i balgarskoto uchastie $\mathrm{v}$ nego [The EUROJOS Project of the Polish Academy of Sciences and the Bulgarian Participation in it]. - Ezik i literatura, № 1-2, s. 175-178.; online: http://www.eziki-literatura.eu/2017/1-2/14-Puhalewa,Sotirov-BG.pdf.

Kremer 2008: Kremer, L. Evropeyskoto pravo na okolnata sreda [European Environmental Law]. Sofia, Siela.

Pernishka 2010: Pernishka, E., D. Blagoeva, S. Kolkovska. Rechnik na novite dumi $\mathrm{v}$ balgarskiya ezik (ot kraya na XX i nachaloto na XXI v.) [Dictionary of New Words in Bulgarian (from the End of the $20^{\text {th }}$ and the Beginning of the $21^{\text {st }}$ Century)]. Sofia, Nauka i izkustvo.

Puzynina 1991: Puzynina, J. Jak pracowac nad jçzykiem wartosci. - In: Jçzyk a kultura, t. 2. Zagadnienia leksykalne i aksjologiczne, red. J. Puzynina, J. Bartminski, Wroclaw, Uniwersytet Wroclawski, s. 129-136.

Puzynina 1992: Puzynina, J. Jçzyk wartosci. Warszawa, Wydawnictwo Naukowe PWN.

Radeva 2007: Radeva, V. Balgarski talkoven rechnik [Bulgarian Explanatory Dictionary]. Plovdiv, Hermes.

Vol'f 1985: Vol'f, E. M. Funktsional'naia semantika otsenki. Moskva, Nauka.

Wierzbicka 1997: Wierzbicka, A. Understanding Cultures through their Key Words: English, Russian, Polish, German, and Japanese. Oxford University Press.

Iliana Genew-Puhalewa, dr hab University of Warsaw Institute of Western and Southern Slavic Studies

Krakowskie Przedmiescie 26/28

00-927 Warszawa, Poland 